Assessment Scale utility in practice in Tartu University Hospital.

Methods: The research method was quantitative, empirical and descriptive, was carried out in Tartu University Hospital in the cardiac surgery, in the paediatric surgery and in the paediatric intensive care unit from 25 June to 23 September 2013. Participants $(n=21)$ used the scale for assessing the infants postoperative pain during three months and gave feedback about the scale utility in practice. An anonymous questionnaire was used to collect the data. The data was analysed using descriptive statistics and Fishers' test.

Results: The results showed the scale is useful for nurses' pain knowledge, improving the nurses' pain knowledge about identification of pain and pain documentation. It is suitable for teaching material to improve the nurses and student nurses' pain knowledges. However, it is not user friendly, is time consuming and completion requires considerable work.

\title{
PO13 - Humanity in the neonatal intensive care unit
}

Inger Jorun Danielsen (Norway) ${ }^{1}$; Ingjerd Gare Kymre (Norway) ${ }^{2}$

${ }^{1}$ UiT The Arctic University of Norway; ${ }^{2}$ University of Nordland

Theme: Ethical issues: dignity and humanity.

Keywords: Communication, developmental support, humanity, developmental support, interaction NICU nursing.

Nurses have a key role in delivering care based on developmental support where proximity and comfort is essential. Our work studies interactions between nurses, infants and parents.

1. Observations and interviews with 23 nurses - qualitative content analysis.

2. Interviews with 18 nurses - phenomenological descriptive analysis.

Five approaches were uncovered: (1) communication with the infant through senses, especially through hands; (2) grasping the infant's condition by combining sensory signs with measurable parameters; (3) promoting the infant's personality and integrity; (4) giving the infant access to parent and opposite; (5) enacting skin-to-skin care.

Humanity as supporting the infant's needs requires understanding the particular infant's cues and signals. There is a need to acknowledge nurses perceptions of SSC as a powerful mutual experience, which is existentially crucial while simultaneously laying the ground for developmental advantages. The two works call for an acknowledgement of the need for parents to be available to premature newborns.

\section{P014 - Paediatric post-operative pain assessment in day surgery}

\section{Emanuela Tiozzo Massimiliano Raponi (Italy) ${ }^{1}$}

${ }^{1}$ Bambino Gesù Children's Hospital, IRCSS, Rome, Italy

Theme: Child protection and managing risk 
Keywords: Children, pain assessment, satisfaction, and smartphone application.

Introduction: There are few studies that describe the usage of applications for pain assessment in the paediatric field. A pain cut-off $\geq 4$ is considered.

Objectives: To evaluate prevalence, incidence and intensity of paediatric pain at home at 24 hours, 5 days and 30 days post-surgery.

Methods: Post-operative pain was monitored with an app developed specifically for this project.

Results: In a population of 214 participants, at 24 hours the prevalence pain evaluation is $71 \%$ (intensity mean 2.3), at 5 days $48 \%$ (intensity mean 1.4 ) and at 30 days $24 \%$ (intensity mean 0.5). The incidence at 24 hours is $73 \%$, at 5 days $5 \%$, at 30 days $2 \%$.

Discussion: The pain app is a useful tool for pain measurement at home, facilitating monitoring and communication between parents and healthcare providers.

Conclusions: The pain application can be a valuable monitoring and decision-making tool for pain management.

\title{
PO15 - PBLS and primary school teachers: survey on training needs in first aid environment
}

\author{
Maria Cristina Tonello (Italy)1; Anna Persico (Italy)2; \\ Giovanni Borrelli (Italy)1 \\ ${ }^{1}$ University of Turin; ${ }^{2}$ Univesity of Turin
}

Theme: School health.

Keywords: Early defibrillation, cardiopulmonary resuscitation, lay, paediatric basic life support, school teachers.

Introduction: Only 15\% of European cases of CPR are performed by a witness. Prompt interventions can increase the person's chances of survival. Secular training programs are gaining even more importance.

Objective: Investigate primary school teachers' level of CPR training in two provinces of Piedmont (Italy).

Methods: We interviewed 667 teachers of which $82 \%$ took part in the study. A questionnaire of 24 questions was created to assess the level of expertise.

Results: 25 teachers had witnessed a student's cardiopulmonary arrest. Just 2 were able to perform a CPR on them. 38\% of the survey participants had been trained in PBLS. $87 \%$ of the educators stated they would not be capable of using an AED and $58 \%$ of them declared their schools are not equipped with AEDs.

Conclusions: This study shows how the majority of the teachers are not adequately trained and it proves the positive effect of a PBLS-D course. 